\title{
Nuclear Segmentation of Glioblastoma Multiforme Cells by Multireference Level Set
}

\section{Rujuta O* and Vyavahare AJ}

Department of Electronics \& Communication, M.E. E \&TC Modern College of Engineering, Pune, India

\begin{abstract}
Histological tissue section consists of rich information about cell type, cellular morphology, cell state and health etc. which is very important for clinical diagnosis and therapy. Automated analysis provides insights of tumor subtypes. Since tumor sections are collected from different laboratory, some issues arises called technical and biological variations. In this paper we developed an approach for nuclear segmentation on tumours histological section, which addresses problems of processing tissues at different laboratory under microscope. Eventually, the resolution is formulated in multi reference level set frame. Experimental results show performance of proposed method.
\end{abstract}

Keywords: Tumor histology sections; Laplacian of gaussian; Gaussian mixture model; Multi reference level set; Nuclear segmentation

\section{Introduction}

The histological tumor tissue section provides morphometric composition to gain insight into cellular morphology, organization, and sample tumor heterogeneity in large cohort. Tumor section represents, strong incorporation and representation to detect mitotic cell, cellular abnormality, and autoimmune responses. If tumor tissue morphology and infrastructure can be marked on a very large scale record set, then it will shows the path for composing prognostic database. The same manner genome analysis techniques identified molecular subtype. Genome-wide molecular analysis (e.g. transcriptome) has the benefits of supervised methods for data examination and pathway quality improvement, which can allow assumption generation for the prevailing techniques. Also histological stains structure are compicated to evaluate due to the biological and technical variations. They provides details understanding into tissue composition and heterogeneity in case of unusual events. The histological tissue section visualized with hematoxylin and eosin stains (H\&E), called as DNA content (e.g. nuclei) and protein respectively in different variation of color. A trained pathologist can detect outline of the rich content in cellular morphology, such as cell shape, cell organization, cell state and health, and cellular production. The main issue to process with the large collection of dataset is color composition concerned to (e.g. damage) and biological variation (e.g. cell type, cell state) across tissue section while scanning under microscope. Regarding to heterogeneity in tumor architecture, nuclear color in the area identified in one tissue area may be same as to cytoplasmic color in another tissue. At the same time, nuclear color density or chromatin content may vary within slide image. Therefore, image research should be supportable and strong, with respect to change in sample arrangements and tumor base within the entire slide image and across the tumor dataset. The tissue parts are scanned at either at $20 \mathrm{X}$ or $40 \mathrm{X}$. Each image is segmented into $1 \mathrm{k} \times 1 \mathrm{k}$ pixels for evolving. This paper includes 1) pre-processing for edge detection.

\section{Related Work}

The main complications in nuclear segmentation are cellular structure (technical, biological) variation or heterogeneity. The techniques for automatic detection and segmentation are based on adaptive thresholding by morphological operator [1,2], fuzzy clustering [3], level set based on gradient information and energy minimization function $[4,5]$. Color deconvolution after optimum thresholding [6], color and texture based hybrid analysis followed by clustering [7]. There is some application combined by above mentioned techniques. i.e., iterative radial voting [8] was used to estimate seeds for the location of nuclei and the model interaction between neighboring nuclei with multiphase level set $[9,10]$, and in [1] an initial segmentation of nucleus with graph cut is obtained by multi scale detection and result further refined with next iteration of same method. Nuclear segmentation through color decomposition, using the same techniques developed for fluorescence microscopy [11]. Still it is a challenging to effectively address the systematical requirements of tumor histological characterization. Thresholding and clustering re-applicable for only constant chromatin content for nuclei in the image. But due to wide variation in chromatin contents problems occurs with overlapping and clumping of the nuclei, also due to tissue thickness, they cannot be segmented properly. The method proposed in [12] focus iterative radial voting on delineation of overlapping nuclei [6], but seed detection can have failed in the presence of wide variation in the nuclear size, lead to fragmentation.

\section{Pre-Processing}

Our method expressed a preprocessing construction representation of nuclear and background of an image based on nuclear response and image denoising using LoG operator nuclear channel.

These representations expressed in terms of GMM and we will then utilize a level set framework to segment foreground and background content. Finally delineated blobs are subjected to convexity constraints for partitioning clumps of nuclei.

\section{Nuclear response LoG filter}

As discussed previous, while processing tumor tissues under different laboratories under microscope, Hence local variations in

*Corresponding author: Rujuta O, Department of Electronics \& Communication, M.E. E \&TC, Modern College of Engineering, Pune, India, Tel: 0202569 6064; E-mail: rujuozarkar88@gmail.com

Received: April 26, 2017; Accepted: May 08, 2017; Published: May 15, 2017

Citation: Rujuta O, Vyavahare AJ (2017) Nuclear Segmentation of Glioblastoma Multiforme Cells by Multireference Level Set. J Bioengineer \& Biomedical Sci 7: 226. doi: 10.4172/2155-9538.1000226

Copyright: @ 2017 Rujuta O, et al. This is an open-access article distributed under the terms of the Creative Commons Attribution License, which permits unrestricted use, distribution, and reproduction in any medium, provided the original author and source are credited. 
an image are captured by LoG filter. LoG is used for edge detection of nuclei and background samples. We calculated MSE, SNR and PSNR for noise level. Normally these parameters are used for image compression but here we used to compare edge detection quality. If the PSNR value is getting less then it shows high edge detection capability. Given dataset scanned at $20 \times$ or $40 \times$ objective during processing each image is manually segmented and processed with LOG filter, and MSE, SNR, PSNR are collected. These tissue sections processed and scanned under different laboratories, some biological variations may occur due to addition of noise. For pre-processing of image denoising is necessary. Here MSE (mean squared error) is used to represent cumulative squared error between detected and original image and PSNR (peak signal to noise ratio) represent a measure of the peak error [13] (Table 1).

\section{Proposed Method}

\section{Gaussian mixture model}

GMM [14] is used to approximate complicated distribution of output coming from object and background of an image, which provide general frame work to characterize heterogeneity. Statistically, a mixture model is usually defined as probability distribution of convex combination of several independent components with different probability distribution. The aim is to estimate from which source output is generated as well as parameter describing source components of GMM. With a set of $N$ samples (image points) from $\mathrm{n}$ dimensional space, $X=\{x 1, \ldots x j, \ldots x N\}$ in which each sample drawn from $M$ Gaussian components. A GMM can be denoted as,

$$
P(X \mid \theta)=\sum_{i=1}^{M} \alpha_{i} p_{j}\left(X \mid \theta_{i}\right) .
$$

where the parameters are $\Theta=\left\{\alpha_{1}, \ldots, \alpha_{M}, \theta_{1}, \ldots \theta_{M}\right\}$ such that, $\sum \alpha \mathrm{i}=1$ and $\alpha_{\mathrm{i}}$ is refers to probability of each component; $\theta_{\mathrm{i}}=\left(\mu_{\mathrm{i}} \sum_{\mathrm{i}}\right)$, $\mu_{\mathrm{i}}$ is mean and $\sum_{\mathrm{i}}$ is covariance matrix $\mathrm{i}=1, . . \mathrm{M}$ Gaussian $\mathrm{x}_{\mathrm{j}}$ is drawn from the probability of $\mathrm{x}_{\mathrm{j}}$ coming from $\mathrm{i}$-th Gaussian is:

$$
\mathrm{p}\left(x_{j} / y_{j}=i, \theta\right)=\frac{\exp \left(-\frac{1}{2}\left(x_{j}-\mu_{i}\right)^{T} \sum_{i}^{-1}\left(x_{j}-\mu_{i}\right)\right)}{2 \pi^{\pi / 2}\left|\sum_{i}\right|^{\frac{1}{2}}}
$$

is used to estimate hidden distribution given data.

The main parameter scale for LoG filter $\sigma$ determined based on dimensions of malignant and normal nuclear size at $20 \times$. Minimum scale value for LoG filter is $\sigma_{\min }$ and maximum scale value is $\sigma_{\text {max }}$ which is defined as expected range of sizes of nuclei (Table 2).

\section{Multireference level set mmodel for nuclei segmentation}

Energy function can be minimized as follows $[15,16]$ :

\section{Let}

1) $\mathrm{F}^{\mathrm{k}}(\mathrm{p})$ is $\mathrm{k}^{\text {th }}$ feature of pixel $\mathrm{p}$; then

2) $\mathrm{P}_{\mathrm{F}}^{\mathrm{k}}$ and $\mathrm{P}_{\mathrm{B}}^{\mathrm{k}}$ are probability of $\mathrm{f}^{\mathrm{k}}$ obtained by nuclei and background,

$$
\mathrm{P}_{\mathrm{F}(\mathrm{p})}^{\mathrm{K}}=\mathrm{GMM}_{\mathrm{F}}^{\mathrm{k}}(\mathrm{p}) / \mathrm{GMM}_{\mathrm{F}}^{\mathrm{k}}(\mathrm{p})+\mathrm{GMM}_{\mathrm{B}}^{\mathrm{k}}(\mathrm{p}) \text { and, }
$$$$
\mathrm{P}_{\mathrm{B}(\mathrm{p})}^{\mathrm{K}}=\mathrm{GMM}_{\mathrm{B}}^{\mathrm{k}}(\mathrm{p}) / \mathrm{GMM}_{\mathrm{F}}^{\mathrm{k}}(\mathrm{p})+\mathrm{GMM}_{\mathrm{B}}^{\mathrm{k}}(\mathrm{p})
$$

3) $\lambda_{\mathrm{k}}$ is weight for $\mathrm{R}_{\mathrm{i}} \lambda_{\mathrm{k}}=<\operatorname{hist}\left(\mathrm{R}_{\mathrm{k}}\right)$,

4) DI is nuclear channel

5) C denotes curve.

$\mathrm{E}=\mu$. Length $(\mathrm{C})+v$ Area(inside $(C))$

\begin{tabular}{|c|c|c|c|}
\hline IMAGE ID & SNR & PSNR & MSE \\
\hline 01 & 12.78 & 19.22 & 58.50 \\
\hline 02 & 14.41 & 18.27 & 48.54 \\
\hline 03 & 13.12 & 18.95 & 56.25 \\
\hline 04 & 14.37 & 18.30 & 48.75 \\
\hline 05 & 15.24 & 17.75 & 44,07 \\
\hline 06 & 15.01 & 18.77 & 42.78 \\
\hline 07 & 15.91 & 17.99 & 40.82 \\
\hline 08 & 13.91 & 18.83 & 51.40 \\
\hline 09 & 15.19 & 18.60 & 44.35 \\
\hline 10 & 14.34 & 18.76 & 48.89 \\
\hline 11 & 13.51 & 18.84 & 53.79 \\
\hline 13 & 16.38 & 18.79 & 38.64 \\
\hline
\end{tabular}

Table 1: Calculation of SNR, PSNR and MSE after processing by LoG filter.

\begin{tabular}{|c|c|c|c|}
\hline \multirow{2}{*}{ IMAGE ID } & \multicolumn{3}{|c|}{ PERFORMANCE MEASURE FOR $\sigma$} \\
\cline { 2 - 4 } & 2.0 & 4.0 & 6.0 \\
\hline 1 & 0.50 & 0.69 & 0.76 \\
\hline 2 & 0.44 & 0.59 & 0.67 \\
\hline 3 & 0.63 & 0.74 & 0.78 \\
\hline 4 & 0.69 & 0.73 & 0.76 \\
\hline 5 & 0.60 & 0.75 & 0.77 \\
\hline 6 & 0.72 & 0.76 & 0.76 \\
\hline 7 & 0.77 & 0.78 & 0.78 \\
\hline 8 & 0.72 & 0.78 & 0.78 \\
\hline 9 & 0.75 & 0.76 & 0.76 \\
\hline 10 & 0.71 & 0.76 & 0.76 \\
\hline 11 & 0.55 & 0,65 & 0.76 \\
\hline 12 & 0.78 & 0.78 & 0.78 \\
\hline 13 & 0.18 & 0.30 & 0.59 \\
\hline
\end{tabular}

Tabel 2: Performance measurement after reducing cross validation error for the range of $\sigma$ ( $\mathrm{min}$ and max scale of LoG based on dimensions of malignant and normal of size of nuclei).

$$
\begin{aligned}
& +\lambda_{F} \int_{F}^{\square}\left|D I(P)-C_{F}(P)\right|^{2} d p \\
& +\lambda_{B} \int_{B}^{\square}\left|D I(P)-C_{B}(P)\right|^{2} d p \\
& -\sum_{k=1}^{N} \lambda^{k} \int_{F}^{\square} \log p_{F}^{k}\left(f^{k}(p)\right) d p-\sum_{k=1}^{N} \lambda^{k} \int_{B}^{\square} \log p_{B}^{k}\left(f^{k}(p)\right) d p \\
& -\alpha \sum_{k=N+1}^{2 N} \lambda^{k-N} \int_{F}^{\square} \log p_{F}^{k}\left(f^{k}(p)\right) d p \\
& -\alpha \sum_{k=N+1}^{2 N} \lambda^{k-N} \int_{B}^{\square} \log p_{B}^{k}\left(f^{k}(p)\right) d p
\end{aligned}
$$

Where, $\mu, v, \lambda_{\mathrm{F}}, \lambda_{\mathrm{B}}$, $\alpha$ are fixed coefficients. $\mathrm{C}_{\mathrm{F}}, \mathrm{C}_{\mathrm{B}}$ are mean intensities of nuclear region i.e., nuclear foreground and background respectively, measured in (DI). The first two terms used for smoothness of nuclear boundary and size respectively. Second two terms for variation in groups of nuclear staining space for nuclear and background respectively. And last four terms related to prior knowledge $[17,18]$.

The separation of nuclei foreground and background can be achieved by minimizing energy function via level set evolution. Regularized Heaviside function as follows:

$$
H(z)=\frac{1}{2}\left(1+\frac{2}{\pi} \arctan \left(\frac{z}{\epsilon}\right)\right)
$$


Citation: Rujuta O, Vyavahare AJ (2017) Nuclear Segmentation of Glioblastoma Multiforme Cells by Multireference Level Set. J Bioengineer \& Biomedical Sci 7: 226. doi: 10.4172/2155-9538.1000226

Page 3 of 4

where $\in$ is regulation parameter of Heaviside function. And delta function as follows:

$$
\delta(Z)=\frac{d}{d z} H(Z)
$$

The impartial energy function can be rewritten as,

$$
\begin{aligned}
& E=\mu \int_{\Omega}^{\square}|\nabla H(\phi(P))| d p+v \int_{\Omega}^{\square}|H(\phi(P))| d p \\
& +\lambda_{F} \int_{\Omega}^{\square}\left|D I(\mathrm{P})-\mathrm{C}_{\mathrm{F}}(P)\right|^{2} \cdot H(\phi(P)) d P \\
& +\lambda_{B} \int_{\Omega}^{\square}\left|D I(\mathrm{P})-\mathrm{C}_{B}(P)\right|^{2} \cdot[1-H(\phi(P))] d P \\
& -\sum_{k=1}^{N} \lambda^{k} \int_{\Omega}^{\square} \log p_{F}^{k}\left(f^{k}(p)\right) d p \cdot H(\phi(p)) d p \\
& -\sum_{k=1}^{N} \lambda^{k} \int_{\bigsqcup_{\Omega}}^{\square} \log p_{B}^{k}\left(f^{k}(p)\right) d p \cdot[1-H(\phi(p))] d p \\
& -\alpha \sum_{k=N+1}^{2 N} \lambda^{k-N} \int_{\Omega}^{\square} \log p_{F}^{k}\left(f^{k}(p)\right) d p \cdot H(\phi(p)) d p \\
& -\alpha \sum_{k=N+1}^{2 N} \lambda^{k-N} \int_{\Omega}^{\square} \log p_{F}^{k}\left(f^{k}(p)\right) d p \cdot[1-H(\phi(p))] d p
\end{aligned}
$$

Energy function minimization achieved by gradient decent method, and Eular Lagrange equation for $\phi$ is:

$$
\begin{aligned}
& \frac{\partial \phi}{\partial t}=\delta(\phi)\left(\mu \cdot \operatorname{div} \frac{\nabla \phi}{|\nabla \phi|}-v\right) \\
& +\delta(\phi)\left(\lambda_{B}\left|D I-C_{B}\right|^{2}-\lambda_{F}\left|D I-C_{F}\right|^{2}\right) \\
& +\delta(\phi)\left(\sum_{k=1}^{N} \log \frac{p_{F}^{k}\left(f^{k}\right)^{\lambda^{k}}}{p_{B}^{k}\left(f^{k}\right)^{\lambda^{k}}}+\sum_{k=N+1}^{2 N} \log \frac{p_{F}^{k}\left(f^{k}\right)^{\alpha \lambda^{k-N}}}{p_{B}^{k}\left(f^{k}\right)^{\lambda^{k-N}}}\right)
\end{aligned}
$$

Basically MRL is region based active contour model and it is not sensitive to initialization. In our approach we initialize zero level set contour at the center of the image having constant radius, which evolved until the differences in spatial location between two zero level set from consecutive iterations are below the threshold (Figure 1).

\section{Nuclear partition via geometric reasoning}

Final part is nuclei partitioning. However after level set evolution, we got binarized image of clumps of nuclei, next step is partition them into single nucleus. Generally nulear shape is convex in shape therefore overlapping nuclei detected by concavities has to be separate out through
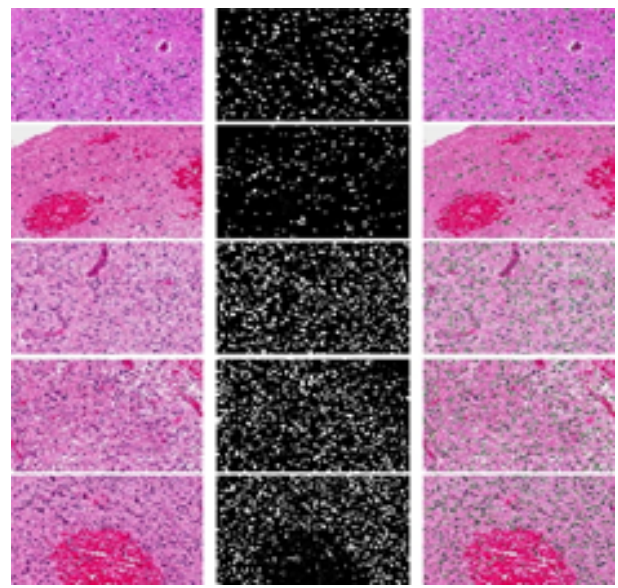

Figure 1: Segmentation result based on MRL approach (a) original images (b) nuclear classification via MRL.

\begin{tabular}{|c|c|c|c|}
\hline IMAGE ID & $\begin{array}{c}\text { No. of cells } \\
\text { detected }\end{array}$ & $\begin{array}{c}\text { Correctly } \\
\text { Segmented }\end{array}$ & Precision \\
\hline 1 & 713 & 165 & 0.92 \\
\hline 2 & 1104 & 265 & 0.94 \\
\hline 3 & 1463 & 341 & 0.93 \\
\hline 4 & 1124 & 238 & 0.85 \\
\hline 5 & 1528 & 337 & 0.88 \\
\hline 6 & 748 & 170 & 0.90 \\
\hline 7 & 1583 & 367 & 0.92 \\
\hline 8 & 2143 & 398 & 0.74 \\
\hline 9 & 1546 & 283 & 0.73 \\
\hline 10 & 1728 & 367 & 0.84 \\
\hline 11 & 602 & 113 & 0.75 \\
\hline 12 & 1694 & 401 & 0.94 \\
\hline 13 & 744 & 168 & 0.90 \\
\hline
\end{tabular}

Table 3: Summary of segmentation performance data for sample images.

geometric reasoning, which is explained by following steps [18]:

1) Detection of maximum point curvature: As the contour of nuclear mask extracted, derivative are computed by derivative of Gaussian.

2) Delauney triangulation: DT is applied to all points of maximum curvature for making possible grouping. The conclusion of grouping then refined by removing edges based on triangulation rules.

3) Geometric reasoning: Properties of both obtained graph and shape of object combined for edge inference.

\section{Discussion}

Our objective dataset consists of hematoxylin and eosin stained GBM tumor section samples collected from different laboratories. Those samples have some technical variation, as they are collected from different laboratories. We manually selected 13 samples and segmentation was carried out on decomposed tissue block.

Gmm component considered are 20. Other parameter setting were $\alpha=1.5, \mu=0.2 /$ timestep, $\lambda=0.05$, and $\sigma\{2.0,4.0,6.0\}$, in which determination of $\sigma$ based on dimensions of malignant and normal nuclear size at $20 \times$, and all other parameter selected to minimize the cross validation error. The algorithm implemented in Matlab 2014.

\section{Conclusion and Future Work}

System can better characterize with variation in data, thus much robust and effective. The LoG filter response gives edge detection information hence background contents are excluded which leads to increase in precision but the drawback of it is the tiny fragments are also indicated. Segmentation performance is indicated by Table 3. Our future work will focus on improving nuclear segmentation by withdrawing the drawbacks and evaluating the method on other remaining tumor types.

\section{Acknowledgement}

The author wish to thank Prof. A.J.Vyavahare for her valuable guidance.

\section{References}

1. Al Kofahi Y, Lassoued W, Lee W, Roysam B (2010) Improved automatic detection and segmentation of cell nuclei in histopathology images. IEEE Trans Biomed Eng 57: 841-852.

2. Phukpattaranont $P$, Boonyaphiphat $P$ (2007) Color based segmentation of nuclear stained breast cancer cell images. ECTI Trans Electr Eng Commun 5: 158-164. 
Citation: Rujuta O, Vyavahare AJ (2017) Nuclear Segmentation of Glioblastoma Multiforme Cells by Multireference Level Set. J Bioengineer \& Biomedical Sci 7: 226. doi: 10.4172/2155-9538.1000226

3. Ballaro B, Florena A, Franco V, Tegolo D, Tripodo C (2008) An automated image analysis methodology for classifying megakaryocytes in chronic myelprliferative disorders. Med Image Anal 12: 703-712.

4. Latson L, Sebek N, Powell K (2003) Automated cell nuclear segmentation in color images of hematoxylin and eosin-stained breast biopsy. Anal Quant Cytol Histol 26: 321-331.

5. Fatakdawala H, Xu J, Basavanhally A, Bhanot G, Ganesan S (2010) Expectation- maximization-driven geodesic active contours with overlap resolution (emagacor): Application to lymphocyte segmentation on breast cancer histopathology. IEEE Trans Biomed. Eng 57: 1676-1690.

6. Chang H, Defilippis R, Tlsty T, Parvin B (2009) Graphical methods for quantifying macromolecules through bright field imaging. Bioinf 25: 1070-1075.

7. Datar M, Padfield D, Cline H (2008) Color and texture based segmentation of molecular pathology images using HSOMS. Proc Int Symp Biomed Imag. pp: 292-295

8. Parvin B, Yang Q, Han J, Chang H, Rydberg B, et al. (2007) Iterative voting for inference of structural saliency and characterization of subcellular events. IEEE Trans Image Process 16: 615-623.

9. Nath S, Palaniappan K, Bunyak F (2006) Cell segmentation using coupled level sets and graph-vertex coloring. In Med Image Comput Computed-Assist Intervention-MICCAI, pp: 101-108.

10. Chang H, Parvin B (2010) Multiphase level set for automated delineation of membrane-bound macromolecules. In Proc Int Symp Biomed Imag, pp: 165-168.
11. Coelho L, Shariff A, Murphy R (2009) Nuclear segmentation in microscope cell images: A hand-segmented dataset and comparison of algorithms. In Proc Int Symp Biomed Imag 5193098: 518-521.

12. Glotsos D, Spyridonos P, Cavouras D, Ravazoula P, Dadioti P (2004) Automated segmentation of routinely hematoxylin-eosin stained microscopic images by combining support vector machine, clustering and active contour models. Anal Quant Cytol Histol 26: 331-340.

13. Gregory RJ, Taraz EB, Devin PS, Gustavo KR, Robert FM (2015) Joint modeling of cell and nuclear shape variation. In Molecular biology of cell 26: 4046-4056.

14. Lei H, Rodney LL, Sameer A, George RT (2010) Local and global gaussian mixture models for hematoxylin and eosin stained histology image segmentation. IEEE Trans. Image process $10^{\text {th }}$ international conference on hybrid intelligent system.

15. Chang H, Han J, Spellman PT, Parvin B (2012) Multireference level set for the characterization of nuclear morphology in glioblastoma multiforme. IEEE Trans Biomed Engineering 59: 3460-3467.

16. Breiman L, Adele C (2001) Random forests. Mach Learn 45: 5-32.

17. Chang H, Fontenay G, Han J, Cong G, Baehner F (2011) Morphometric analysis of TGCA gliobastoma multiforme. BMC Bioinf 12: 484.

18. Bunyak F, Hafiane A, Palanippan K (2011) Histopathology tissue segmentation by combining fuzzy clustering with multiphase vector level set. Adv Exp Med Biol 696: 413-424. 